\section{Revista Mexicana de Astronomia y Astrofisica}

Revista Mexicana de Astronomía y Astrofísica ISSN: 0185-1101

rmaa@astroscu.unam.mx

Instituto de Astronomía

México

Relaño, M.; Peimbert, M.; Beckman, J. E.

Photoionization models of NGC 346

Revista Mexicana de Astronomía y Astrofísica, vol. 12, noviembre, 2002, pp. 263-264

Instituto de Astronomía

Distrito Federal, México

Available in: http://www.redalyc.org/articulo.oa?id=57112108

How to cite

Complete issue

- More information about this article

Journal's homepage in redalyc.org

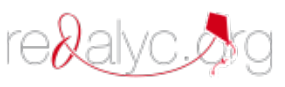

Scientific Information System Network of Scientific Journals from Latin America, the Caribbean, Spain and Portugal Non-profit academic project, developed under the open access initiative 
RevMexAA (Serie de Conferencias), 12, 263-264 (2002)

\title{
PHOTOIONIZATION MODELS OF NGC 346
}

\author{
M. Relaño, ${ }^{1}$ M. Peimbert, ${ }^{2}$ and J. E. Beckman ${ }^{1,3}$
}

We have compared direct estimates of the ionizing photon flux from the stars observed within the H II region NGC 346 in the Small Magellanic Cloud with its observed $\mathrm{H} \alpha$ luminosity using a series of photoionization models of the region. The tests were based on the use of the photoionization code Cloudy (Ferland 2000), aided by $\mathrm{H}, \mathrm{He}, \mathrm{N}, \mathrm{O}, \mathrm{Ne}, \mathrm{S}, \mathrm{Ar}$, and Fe emission line observations of Peimbert, Peimbert, \& Ruiz (2000, hereinafter PPR). A key result is that some $46 \%$ of the Lyman continuum photons escape from the region. The abundances of $\mathrm{N}, \mathrm{O}, \mathrm{Ne}, \mathrm{S}, \mathrm{Ar}$, and $\mathrm{Fe}$ relative to $H$ were determined using the ionization structure of the best model, and are compared with those derived from empirical ionization correction factors.

We estimate directly the photoionization spectrum that will be used for the models based on the spectral classification of the blue stars presented by Massey, Parker, \& Garmany (1989). We assume that these stars are blackbodies emitting at specified effective temperatures. This assumption is plausible since the stars have high $T_{\text {eff }}$. To derive the ionizing flux, we have adopted the stellar parameters presented by Vacca, Garmany, \& Shull (1996) and found that the total ionizing flux amounts to $40.06 \times 10^{39} \mathrm{erg} \mathrm{s}^{-1}$. Using this ionizing luminosity as an input to a homogeneous spherical model for NGC 346 and assuming that all the ionizing photons are absorbed within the nebula, we obtain, using Cloudy a value for the $\mathrm{H} \alpha$ luminosity of the $\mathrm{H}$ II region of $1.78 \times 10^{39} \mathrm{erg} \mathrm{s}^{-1}$.

From the observed $\mathrm{H} \alpha$ flux of $1.55 \times 10^{-9}$ erg s${ }^{-1} \mathrm{~cm}^{-2}$ (Kennicutt \& Hodge 1986), an interestellar absorption correction of $C(\mathrm{H} \alpha) \sim 0.10$ dex (from PPR) and a distance of $64 \mathrm{kpc}$ (Reid 1999) for SMC, the emitted $\mathrm{H} \alpha$ luminosity is $9.55 \times 10^{38}$ $\operatorname{erg~} \mathrm{s}^{-1}$. The difference between the $\mathrm{H} \alpha$ luminosity from the stellar radiation and that observed by

\footnotetext{
${ }^{1}$ Instituto de Astrofísica de Canarias, Tenerife, Spain (mpastor@ll.iac.es).

${ }^{2}$ Instituto de Astronomía, UNAM, México (peimbert@astroscu.unam.mx).

${ }^{3}$ Consejo Superior de Investigaciones Científicas, Spain (jeb@ll.iac.es).
}

Kennicutt \& Hodge implies that $46 \%$ of the ionizing photons escape from NGC 346.

We have implemented different ionization models using Cloudy to reproduce the emission line observations of PPR. The three spherical models are: a constant density model, a clumpy model with a non-zero filling factor, and a clumpy model with two density zones. Plane-parallel clumpy models with a constant density and with two different density regions have also been implemented. All these models are integrated over a volume defined by observed selected slits from PPR and constrained to reproduce the flux derived from observations.

The spherical models cannot fit all the observed line ratios from PPR. The spherical model with two uniform density zones, $n_{1}=140 \mathrm{~cm}^{-3}$ for the inner zone and $n_{2}=50 \mathrm{~cm}^{-3}$ for the outer, agrees well with the observations, although it seems quite difficult to predict [O II] and [O III] simultaneously. Also, while the $I(\lambda 4686) / I(\mathrm{H} \beta)$ ratio in the planeparallel models is underpredicted, the ratio in the spherical models is overpredicted. In all these models, the predicted $I(\lambda 4363) / I(\lambda 5007)$ ratio is smaller than the observed ratio, probably implying the presence of an additional energy source not included in the models.

The total abundances derived from the model ionization structure agree well for $\mathrm{O}, \mathrm{Ne}, \mathrm{S}$, and $\mathrm{Ar}$ with those determined from empirical ICF predictions. Alternatively the $\mathrm{N}$ abundance derived from the models is a factor of two higher than that derived from an empirical ICF prediction, this problem needs to be studied further. The $\mathrm{Fe} / \mathrm{O}$ ratio is a factor of three smaller than in the Sun, which probably indicates that most of the iron atoms are embedded in dust grains.

\section{REFERENCES}

Ferland, G. J. 2000, RevMexAA(SC), 9, 153

Kennicutt, J. R. \& Hodge, P. 1986, ApJ 306, 130

Massey, P., Parker, J. W., \& Garmany, C. 1989, AJ 98, 4

Peimbert, M., Peimbert, A., \& Ruiz, M. T. 2000, ApJ 541, 688 (PPR)

Reid, N. 1999, ARA\&A 37, 191

Vacca, W. D., Garmany, C. D., \& Shull, J. M. 1996, ApJ 460,914 


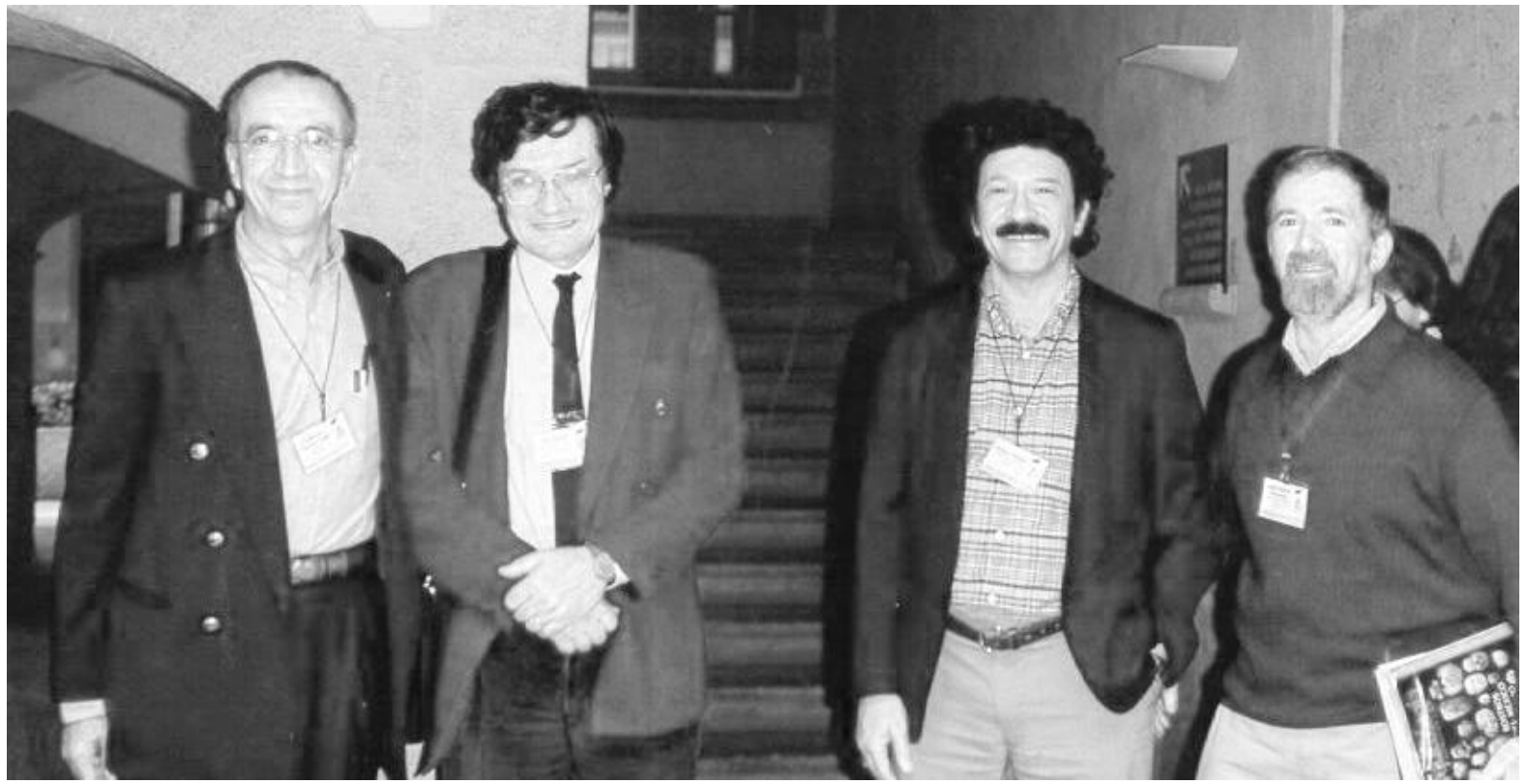

Guillermo Tenorio-Tagle, Jan Palouš, Bob Rubin, and John Beckman 